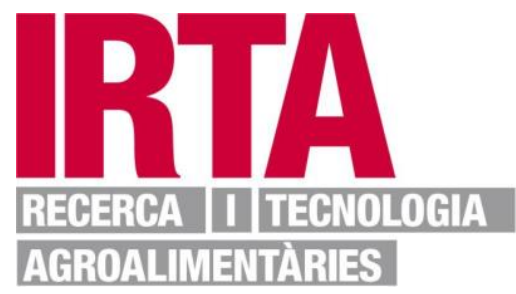

This is the peer reviewed version of the following article: Jin, Jing, Ling Gao, Lan Zhao, Zhong-shan Gao, Xiong-wei Li, Han-bing Xie, and Jun-bei Ni et al. 2019. "Selection Of Pru P 3 Hypoallergenic Peach And Nectarine Varieties". Allergy 75 (5): 1256-1260. doi:10.1111/all.14102, which has been published in final form at https://doi:10.1111/all.14102. This article may be used for non-commercial purposes in accordance with Wiley Terms and Conditions for Use of Self-Archived Versions http://www.wileyauthors.com/self-archiving.

Document downloaded from: 


\section{Selection of Pru p 3 hypoallergenic peach and nectarine varieties}

2 To the Editor,

3 Peach is an important fruit consumed worldwide. However, it is also one of the most

4 frequently reported allergenic fruits ${ }^{1}$. Component diagnosis of peach allergy indicates

5 Pru p 1, Pru p 2, Pru p 3 Pru p 4, Pru p 7 and Pru p 9 are involved ${ }^{2,3}$. Pru p 3 is the

6 dominant allergen responsible for severe allergic reaction ${ }^{4}$ and it is considered to be

7 the primary sensitizer to other LTPs in Mediterranean and Central Europe ${ }^{5}$.

8 The levels of Pru p 3 differ between varieties ${ }^{6}$. To date, measurement of Pru p 3 in a

9 limited number of peach and nectarines from Spain, US and Italy has been reported ${ }^{7}$. Significant variation of allergen concentration in processed foods containing peach has also been observed ${ }^{8}$. The content of Pru p 3 of peach/nectarine determines the potential risk for peach allergic patients.

China is the origin of peach with representative genetic diversity to be explored for hypoallergenic varieties ${ }^{9}$. A core collection of 103 varieties cultivated in Jiaxing, Zhejiang Province were selected to represent this diversity, including 23 nectarines and 80 peach varieties (with fruit hair, round or flat, 77 cultivated, three wild) (Supplementary Table 1). The soluble solid content (SSC), ripening date and peach aroma intensity were recorded. Specific methods are detailed in the Supporting Information. Pru p 3 was quantified by ELISA based on our previous research ${ }^{6}$.

Significant differences in Pru p 3 content were identified in individual varieties $(P<0.0001)$ (Figure 1A). Most nectarine varieties had low Pru p 3 content with pedigree of 'Armking' and 'Mayfire' (Supplementary Table 1), while a large variation was observed in peach: the lowest $(3.5 \mu \mathrm{g} / \mathrm{g})$ in a wild peach, and the highest $(64.4 \mu \mathrm{g} / \mathrm{g})$ in flavorsome yellow flesh peaches. In cultivated peach and nectarine, the level was higher than in wild varieties, and usually higher in peach than nectarine. In addition, fruit harvest month greatly influenced the Pru p 3 content $(P<0.001)$. Peach varieties ripening late generally had higher levels than earlier ones: $40.19 \mu \mathrm{g} / \mathrm{g}$ on average for varieties ripening in August/September, about three times the level of 
those in May (Figure 1B). Fruit flesh color also reflected the Pru p 3 content $(P=0.0072, \mathrm{n}=100)$. The results showed that hypoallergenic varieties were mainly yellow flesh nectarines and red flesh peaches (Figure 1C, D). Correlation analysis between Pru p 3 and soluble solid content (SSC) and the influence of aroma showed that higher Pru p 3 content related to higher SSC $(P=0.0006, \mathrm{r}=0.3394, \mathrm{n}=98)$ and stronger aroma $(P=0.0002)$ (Figure $1 \mathrm{E}, \mathrm{F})$, indicating that good quality peaches had high allergenic potential. The Pru p 3 content of flat peaches, becoming more popular in Mediterranean countries and China, is expected to be high, as demonstrated in Supplementary Table 2 . There was $4 \%$ to $30 \%$ variation between years (2016-2018) for the same variety. The distribution of Pru p 3 in different parts of peach fruit differs greatly: the content in peel was 13 to 60 times higher than in pulp (Supplementary Table 2).

Immunocytochemical observations of Pru p 3 in four varieties with significantly different Pru p 3 content showed striking differences. In low Pru p 3 content 'Hu You

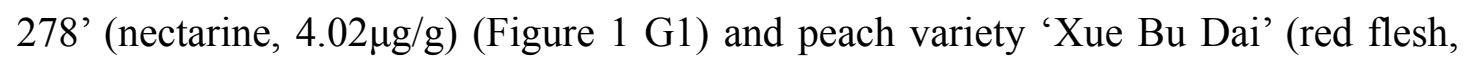
$4.00 \mu \mathrm{g} / \mathrm{g}$, Figure 1G2), small amounts of Pru p 3 was located in the pericarp layer, and less in the mesocarp. In high Pru p 3 content yellow flesh peach variety 'Mei Jin' $(37.42 \mu \mathrm{g} / \mathrm{g}$, Figure 1G3) and 'Jin Shuo' $(57.89 \mu \mathrm{g} / \mathrm{g}$, Figure 1G4), the fluorescent signals were clearly stronger than 'Xue Bu Dai' and 'Hu You 278' in both the pericarp and mesocarp cells. All three peach varieties (with hair) (Figure G2, 3, 4) contained high Pru p 3 in the hair. These results were consistent and data are shown in Supplementary Table 2. The Pru p 3 content in the pulp of a yellow flesh peach cultivar such as 'Jin Shuo', was higher than that in the whole fruit of some nectarines or red flesh peaches. This indicates that, although peeling the fruit is theoretically an effective way to reduce Pru $\mathrm{p} 3$, it is not always practical and does not always alleviate the risk of peach allergic reaction. The correct choice of variety is better.

Skin prick testing (SPT) was performed on nine patients from Shanxi and Zhejiang provinces, recruited on the basis of their clinical history and a positive peach ImmunoCAP. All of them had provided written informed consent and approved by the 
local ethics committee (authorization No. 2011-R-1, Second Affiliated Hospital, College of Medicine, Zhejiang University), in collaboration with the Third People's Hospital of Datong, Shanxi (authorization No. 2015-001). The identified low Pru p 3

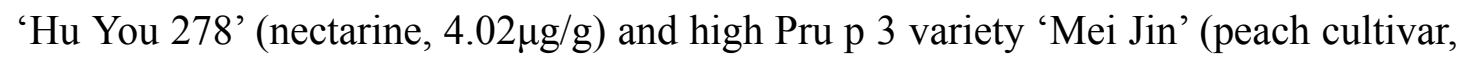
$37.42 \mu \mathrm{g} / \mathrm{g}$ ), according to our quantification and immunocytochemical localization, were tested to assess the sensitization of varieties with different Pru p 3 content in allergic individuals. All nine allergic subjects had a positive SPT to fresh peach cv 'Mei Jin', higher than those with nectarine cv Huyou 278, and one patient was negative to nectarine (Table 1). ImmunoCAP results showed that 9/9 were positive to peach, 7/9 positive to Pru p 3 and /or Art v 3, 2/9 positive to Pru p 4 and none of them positive to Pru p 1 (Table 1).

In summary, Pru p 3 content differed considerably depending on the variety, related to fruit type, flesh color and ripening date. Low risk varieties were nectarines and red flesh peach, maturing in May to July and with low or mild fruit quality. This research provides a directive for evaluating potential Pru p 3 levels for patients and clinical doctors. We identified several hypoallergenic nectarines (May Fire, $\mathrm{Hu}$ You 278) and three red-flesh peach varieties 'Xue Bu Dai', 'Zi Xue Tao' and 'Wu Yue Xian'. Because of the narrow genetic background of nectarines worldwide from limited founder cultivars such as 'Armking' and 'Mayfire', hypoallergenic nectarines are recommended for further clinical trials. 


\section{Legend}

79 Figure 1. Comparison of Pru p 3 content in different variety groups and 80 immunocytochemical localization of Pru p 3 in low and high content varieties.

81 A: Pru $\mathrm{p} 3$ content in different fruit types. B: different harvest month. C: flesh color of nectarines.

82 D: flesh color of peaches. E: Influence of soluble solid content (SSC) and F: aroma intensity. G:

83 Immunocytochemical localization of Pru p 3 in (1) 'Hu You 278', (2)- 'Xue Bu Dai', (3) 'Mei Jin'

84 and (4) 'Jing Shuo'. Scale bar $=200 \mu \mathrm{m}, 100 \mathrm{x}$ magnification. Difference between groups was

85 assessed by Kruskal-Wallis nonparametric test followed by Dunn's multiple comparison test (A, B,

86 D, F) and Kolmogorov-Smirnov test (C). ${ }^{*} \mathrm{p}<0.05,{ }^{* *} \mathrm{p}<0.01,{ }^{* * *} \mathrm{p}<0.001,{ }^{* * * *} \mathrm{p}<0.0001$; ns, not

87 significant. Data expressed as mean \pm SE. 


\section{JIN et al}

88

89

90

91

\section{Reference}

1. Gao ZS, Yang ZW, Wu SD, et al. Peach allergy in China: a dominant role for mugwort pollen lipid transfer protein as a primary sensitizer. J Allergy Clin Immunol, 2013, 131(1), 222-224.

2. Matricardi PM, Kleine-Tebbe J, Hoffmann HJ, et al. EAACI Molecular Allergology User's Guide. Pediatr Allergy Immunol, 2016, 27 Suppl 23:1-250.

3. Somoza ML, Garrido-Arandia M, Victorio-Puche L, et al. Peach tree pollen and Pru p 9 may induce rhinoconjunctivitis and asthma in children. Pediatr Allergy Immunol, 2019, 00:1-4.

4. Ma S, Yin J, and Jiang N. Component-resolved diagnosis of peach allergy and its relationship with prevalent allergenic pollens in China. J Allergy Clin Immunol, 2013, 132:764-767.

5. Mothes-Luksch N, Raith M, Stingl G, et al. Pru p 3, a marker allergen for lipid transfer protein sensitization also in Central Europe. Allergy, 2017, 72:1415-1418.

6. Gao ZS, Ma YT, Zhou X, et al. Quantification of peach fruit allergen lipid transfer protein by a double monoclonal antibody-based sandwich ELISA. Food Anal Methods, 2016, 9(4), 823-830.

7. Ahrazem O, Jimeno L, Lopez-Torrejon G, et al. Assessing allergen levels in peach and nectarine cultivars. Ann Allergy Asthma Immunol, 2007, 99(1), 42-47.

8. Duffort OA, Polo F, Lombardero M, et al. Immunoassay To quantify the major peach allergen Pru $\mathrm{p} 3$ in foodstuffs. differential allergen release and stability under physiological conditions. J Agri Food Chem, 2002, 50(26), 7738-7741.

9. Li XW, Meng X, Jia HM, et al. Peach genetic resources: diversity, population structure and linkage disequilibrium. BMC Genetics, 2013.14(1), 84. 
Author's name

109 Jing $\operatorname{Jin}^{1^{*}}$, Ling Gao ${ }^{1 *}$, Lan Zhao ${ }^{1}$, Zhong-shan Gao ${ }^{1,2,3}$, Xiong-wei $\mathrm{Li}^{4}$, Han-bing $110 \mathrm{Xie}^{1}$, Jun-bei $\mathrm{Ni}^{1}$, Ke-xin $\mathrm{Gan}^{1}$, Shan-dong $\mathrm{Wu}^{2}$, Zheng-wen $\mathrm{Ye}^{4}$, Jun Luo ${ }^{4}, \mathrm{Ke} \mathrm{Cao}^{5}$, 111 Rui-juan $\mathrm{Ma}^{6}$, Miao-jin Chen ${ }^{7}$, Pere Arús ${ }^{8}$, Serge A. Versteeg ${ }^{3}$, Hui-ying Wang 9 , 112 Meiling Liu ${ }^{10}$, Hui-juan Jia ${ }^{1}$, Ronald van Ree ${ }^{3}$

$113{ }^{1}$ College of Agriculture and Biotechnology, Zhejiang University, 310058, Hangzhou, 114 China

$115{ }^{2}$ Allergy Research Center, Zhejiang University, 310058, Hangzhou, China

$116{ }^{3}$ Departments of Experimental Immunology and of Otorhinolaryngology, Amsterdam

117 UMC, University of Amsterdam, Meibergdreef 9, 1105 AZ Amsterdam, the

118 Netherlands

$119{ }^{4}$ Forest \& Fruit Tree Institute, Shanghai Academy of Agricultural Sciences, Shanghai, 120 201403, China

$121{ }^{5}$ Zhengzhou Fruit Research Institute, China Academy of Agricultural Sciences,

122 Zhengzhou, China

$123{ }^{6}$ Horticultural Institute, Jiangsu Academy of Agricultural Sciences, Zhong-Lin Street 12450, Nanjing, 210014, China

$125{ }^{7}$ Fenghua Honey Peach Institute, Xikou, Fenghua, Zhejiang Province, 315521, China $126{ }^{8}$ IRTA, Centre de Recerca en Agrigenòmica CSIC-IRTA-UAB-UB, Campus UAB 127 Edifici CRAG, Cerdanyola del Vallès (Bellaterra), 08193 Barcelona, Spain

$128{ }^{9}$ Department of Allergy, the Second Affiliated Hospital, School of Medicine, Zhejiang 129 University, Hangzhou, 310013, China.

$130{ }^{10}$ Department of Allergy, The Third People's Hospital of Datong, Datong, Shanxi, 131 037008, China

132 *Jing and Gao contributed equally to this research.

133 Correspondence: Prof. Zhongshan Gao, E-mail address: gaozhongshan@zju.edu.cn; 134 and Dr. Xiongwei Li, lixiongweisea@163.com 


\section{Acknowledgements}

This study was funded by the National Natural Science Foundation of China (grant 31272131 and 31372040), Shanghai Science and Technology Committee Rising-Star Program (19QB1404600) and The Key Project for New Agricultural Cultivar Breeding in Zhejiang Province, China (2016C02052-5). Dr. WANG Lirong, Dr. YU Mingliang, Dr. SHEN Zhijun, Dr. NIU Liang, Dr. WANG Zhiqiang, Mr. MA Zhisheng, Mr. LIU Hangkong, Dr. TIAN Jianbao and Mr. WU Dajun provided some peach accessions. Ms WANG Xuefeng and Ms ZHAO Xiuzhen performed the skin prick test. Dr. ZHANG Xianqi and Ms WANG Tingting provided assistance in the sera collection.

\section{Conflict of Interest}

Gao ZS has received grants from National Natural Science Foundation of China (31272131) and the Key Project for New Agricultural Cultivar Breeding in Zhejiang Province (2016C02052-5), Dr. Li XW has received grant from Shanghai Science and Technology Committee Rising-Star Program; Dr. Jia HJ has received grants from National Natural Science Foundation of China (31372040) and the Key Project for New Agricultural Cultivar Breeding in Zhejiang Province (2016C02052-5). The remaining authors declare that they have no relevant conflict of interests.

\section{Authors' contributions}

ZSG, RvR and JHJ conceived and planned the study. JJ, GL, LZ, XWL, HBX and KXG collected peach samples and extracted total proteins. JJ, LG, LZ, JBN, SDW and SAV established ELISA method and data analyses. XWL, KC, ZWY, JL, RJM, KC, MJC, PA and HJJ selected the core peach collection. MLL and HYW performed the diagnosis and skin prick tests. JJ, GL, ZSG, XWL and RvR wrote the manuscript. All authors read and approved the final manuscript.

\section{Keywords}

Allergen; peach; Pru p 3; hypoallergenic varieties; protein quantification 

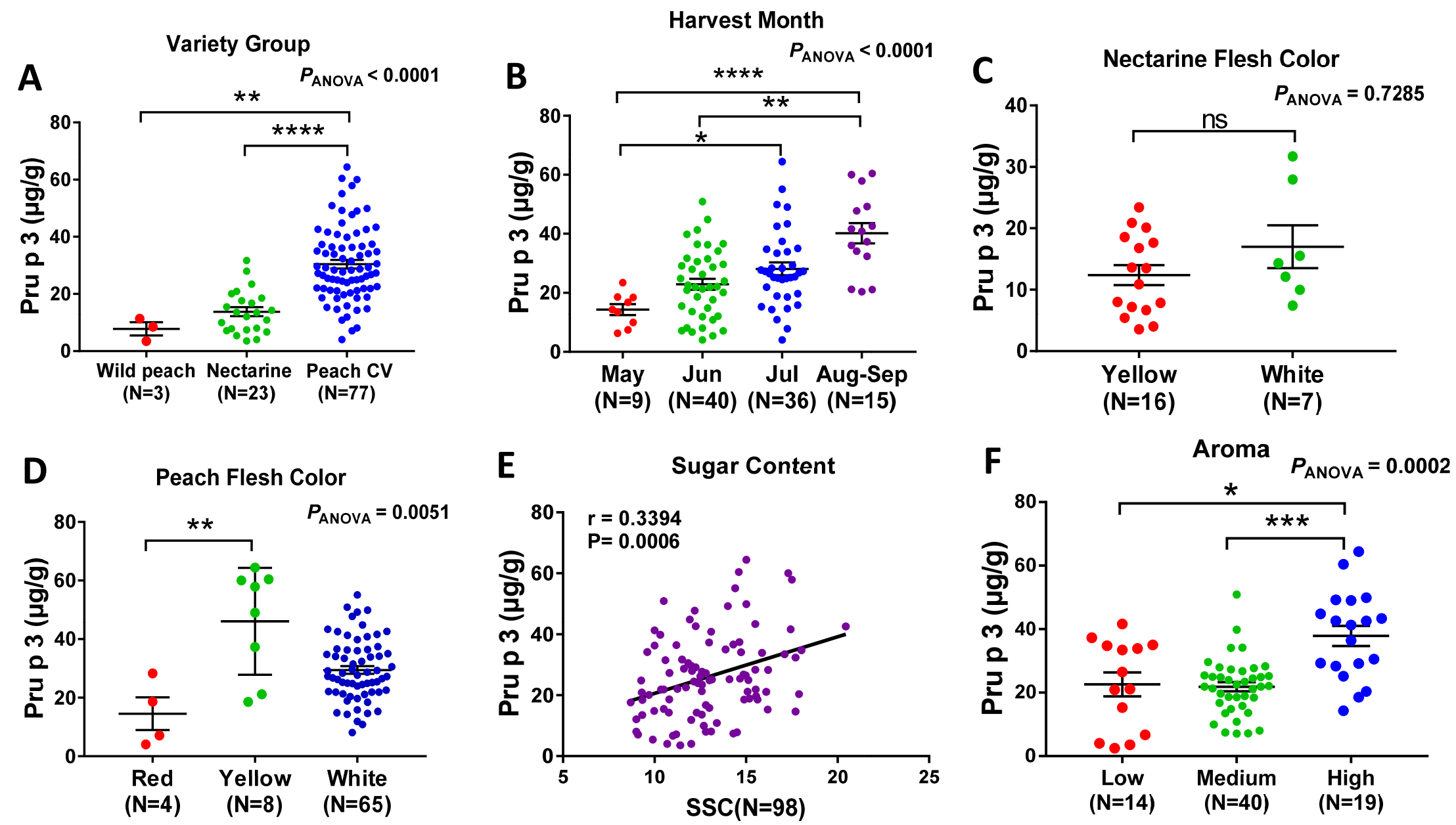

G

(1)

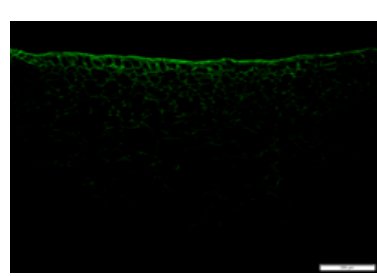

(3)

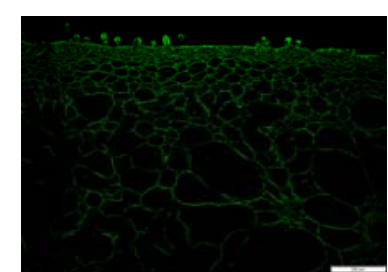

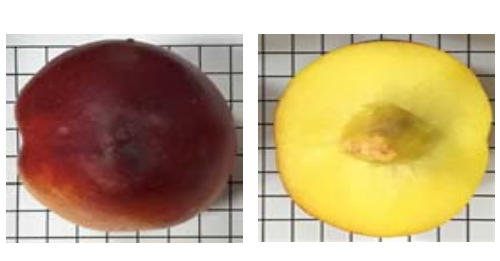

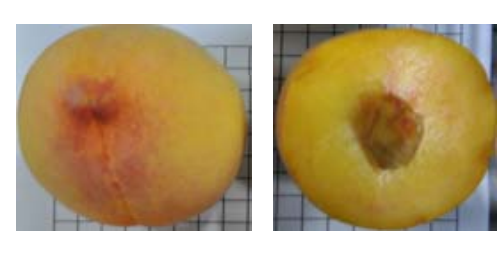

(2)
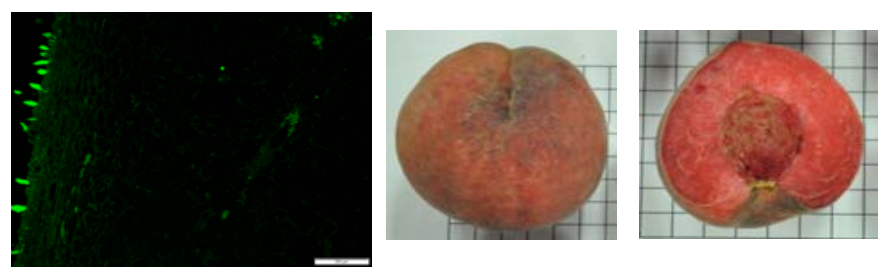

(4)
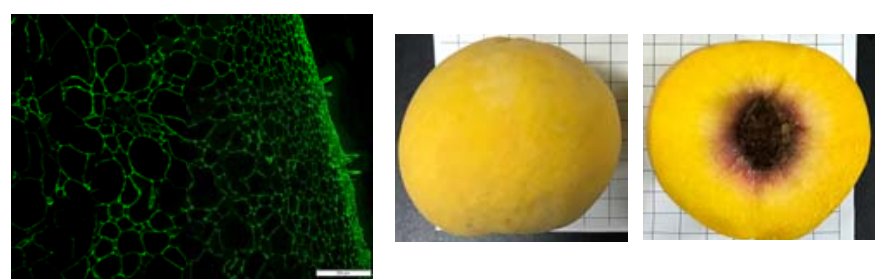


\section{Detailed methods used:}

\section{Preparation of fruit samples}

Soluble solid content (SSC) was measured with a digital refractometer (ATAGO, PR-101 $\alpha$ ), and peach aroma intensity was classified subjectively as light, medium or strong (Supplementary Table 1) based on the Descriptors and Data Standard for Peach ${ }^{1}$. For 24 varieties (Table S2), the peel and pulp were separated: for melting peaches, this can easily be done by hand, while for nectarines a knife peeler was used, with $1 \mathrm{~mm}$ thickness.

\section{Immunocytochemical localization of Pru p 3}

Four varieties 'Hu You 278', 'Xue Bu Dai', 'Mei Jin' and 'Jin Shuo' were used for immuno-cytochemical localization with specific monoclonal antibody 4-1 used in ELISA quantification above. The EnVision two steps method was used for immunocytochemical analysis. Harvested fruits were sectioned and fixed in FAA. After recovering the allergen by boiling in citrate buffer solution (0.01M, pH6.0) for $20 \mathrm{~min}$, sections were exposed to $3 \% \mathrm{H}_{2} \mathrm{O}_{2}$ in methanol for 10 minutes to quench endogenous peroxidase activity, and nonspecific binding was blocked by incubation in PBS containing 5\% BSA for 30 minutes. Peach tissue sections were incubated with anti-Pru p 3 antibody $4-1(1 \mathrm{mg} / \mathrm{ml})$ at a titer of $1: 100$ overnight at $4^{\circ} \mathrm{C}$. After washing, sections were incubated with the secondary antibody labeling with green fluorescence Alexa Fluor 488 goat anti-mouse $\operatorname{IgG}(\mathrm{H}+\mathrm{L})$ for 1 hour, with exposure to a substrate chromogen mixture for 10 minutes. Green color was present on the section viewed under an OLYMPUS DP80 microscope after staining with hematoxylin. Images of sections from different varieties were taken with the same magnification and the same exposure time.

\section{Patients and Skin Prick Test}

Five patients from Shanxi and four from Zhejiang province were recruited on the basis of their clinical history and positive skin tests to peach extracts. All of them had provided the written informed consents. Written consent was obtained from all participants (or their representative) and the study was approved by the local ethics committee (authorization No. 2011-R-1, Second Affiliated Hospital, College of Medicine, Zhejiang University), in collaboration with the Third People's Hospital of Datong, Shanxi (authorization No. 2015-001) A serum sample from each patient was taken during the first visit and was kept frozen at $-40^{\circ} \mathrm{C}$ until used. Skin tests to fresh peach were performed by skin prick test, following the technique described by Dreborg and Foucard ${ }^{2}$.

\section{Reference}

1. Wang, L., Zhu, G. Descriptors and data standard for peach (Prunus persica L.). Beijing: China Agriculture Press. (in Chinese) 2005.

2. Dreborg, S., \& Foucard, T. Allergy to apple, carrot and potato in children with birch pollen allergy. Allergy, 1983, 38(3), 167-172. 
Supplementary Table 1. List of 103 core peach varieties and their basic fruit traits with Pru p 3 content.

\begin{tabular}{|c|c|c|c|c|c|c|c|c|}
\hline Code & Variety & Pedigree & Origin country & Fruit trait* & SSC (\%) & Aroma & Maturity time & Pru p 3 (ug/g FW) \\
\hline 1 & Mao Tao 1 & Wild peach for rootstock & Zhejiang province, China & PWR & 9.4 & $\mathrm{~L}$ & Early August & 3.47 \\
\hline 2 & Xue Bu Dai & Landrace & Henan province, China & PRR & 12.0 & $\mathrm{~L}$ & Early July & 4.00 \\
\hline 3 & Hu You 278 & Rui Guang $3 \times$ May Fire & Shanghai, China & NYR & 10.7 & $\mathrm{~L}$ & Middle June & 4.02 \\
\hline 4 & Hu You 005 & Rui Guang $3 \times$ May Fire & Shanghai, China & NYR & 9.9 & $\mathrm{~L}$ & Middle June & 5.42 \\
\hline 5 & May Fire & Seeding Selection of Armking & USA & NYR & 11.4 & $\mathrm{~L}$ & Late May & 3.54 \\
\hline 6 & Armking & Palomar $\times$ Springtime & USA & NYR & 11.0 & $\mathrm{~L}$ & Early June & 6.68 \\
\hline 7 & Zi Xue Tao & Landrace & Zhejiang province, China & PRR & 9.1 & $\mathrm{~L}$ & Late June & 7.12 \\
\hline 8 & Zhong You Tao 11 & Zhong You Tao 5×SD9238(Rui Guang 3x May fire) & Henan province, China & NYR & 11.2 & M & Late June & 7.18 \\
\hline 9 & Zao Hong Zhu & Jing $\mathrm{Yu} \times \mathrm{A} 369$ & Beijing, China & NWR & 14.3 & M & Late May & 7.41 \\
\hline 10 & Zhong You Tao 8 & Hong Shan $\mathrm{Hu} \times$ Sunshine & Henan province, China & NYR & 14.5 & $\mathrm{~L}$ & Middle July & 7.85 \\
\hline 11 & Wu Yue Xian & Landrace & Shanxi province, China & PRR & 9.0 & $\mathrm{~L}$ & Middle June & 8.02 \\
\hline 12 & Pan Tao Wang & Early Red $2 \times$ Zao Lu Pan Tao & Henan province, China & PWF & 13.1 & M & Early June & 8.13 \\
\hline 13 & Mao Tao 2 & Wild peach for rootstock & Zhejiang province, China & PYR & 11.9 & $\mathrm{~L}$ & Early August & 8.53 \\
\hline 14 & Zhong Nong Jin Hui & Rui Guang $2 \times$ Armking & Henan province, China & NYR & 12.8 & M & Early June & 9.07 \\
\hline 15 & Zhong You Tao 12 & 6-2×SD9238 (Ruiguang $3 \times$ Mayfire) & Henan province, China & NWR & 12.7 & M & Late May & 9.97 \\
\hline 16 & Jin Xia You Pan & Xia Guang $\times$ 'NF' & Jiangsu,China & NYF & 13.4 & $\mathrm{~L}$ & Early July & 10.89 \\
\hline 17 & Mao Tao 3 & Wild peach for rootstock & Zhejiang province, China & PWR & 12.4 & $\mathrm{~L}$ & Middle August & 11.35 \\
\hline 18 & Chun Mei & (Zao Hong $2 \times$ Flatpeach $) \times($ Rui Guang $3 \times$ May Fire) & Henan province, China & PWR & 12.0 & M & Early June & 11.87 \\
\hline 19 & Zhong You Tao 5 & Rui Guang $3 \times$ May Fire & Henan province, China & NWR & 9.0 & $\mathrm{~L}$ & Early June & 12.13 \\
\hline 20 & Chao Li Chun & Ruiguang $3 \times$ Mayfire & Beijing, China & NYR & 9.3 & $\mathrm{~L}$ & Late May & 13.53 \\
\hline 21 & Zhong You Tao 4 & Ruiguang $16 \times$ May Fire & Henan province, China & NYR & 12.6 & M & Early June & 13.62 \\
\hline 22 & Jin Xia & 75-3-9(Okubo ×Okitsu) $\times 75-6-18$ (Okitsu) & Shanxi province, China & NWR & 12.3 & M & Early July & 14.30 \\
\hline 23 & Zao Mei & Qing Feng $\times$ Zhao Xia & Beijing, China & PWR & 10.8 & M & Late May & 14.33 \\
\hline
\end{tabular}




\begin{tabular}{|c|c|c|c|c|c|c|c|c|}
\hline 24 & Jin Yuan & Jin Xiu $\times 75-1-3$ & Shanghai, China & PWR & 17.7 & $\mathrm{~L}$ & Late July & 14.61 \\
\hline 25 & Hakuri & Feicheng Tao & Japan & PWR & 16.2 & $\mathrm{~L}$ & Middle July & 14.84 \\
\hline 26 & Zao Hong Lu & Armking $\times 81-3-3$ & Bejing,China & NWR & 10.5 & M & Early June & 15.50 \\
\hline 27 & Zao Jiu Bao & Bud mutation from Okubo & Shanxi province, China & PWR & 13.0 & M & Early July & 15.82 \\
\hline 28 & Rui Guang 2 & Jingyu x NJN76 & Beijing, China & NYR & 12.8 & M & Late May & 16.77 \\
\hline 29 & Hu You 018 & Rui Guang $3 \times$ May Fire & Shanghai, China & NYR & 8.7 & M & Middle June & 17.64 \\
\hline 30 & Chun Lei & Sunago Wase $\times$ Bai Xiang Lu & Shanghai, China & PWR & 9.3 & M & Late May & 18.43 \\
\hline 31 & Jin Xiang & Bei Nong $2 \times 60-27-7$ & Shanghai, China & PYR & 15.6 & $\mathrm{H}$ & Middle July & 18.58 \\
\hline 32 & Nan Fang Jin Mi & (Sunred $\times$ Maravilha) $\quad 1-15 \times$ Shu Guang & Henan province, China & NYR & 14.9 & M & Late May & 18.59 \\
\hline 33 & Yang Tao & Landrace & Zhejiang province, China & PWR & 10.8 & M & Middle June & 18.62 \\
\hline 34 & Hang Mi 1 Hao & unknown & Zhejiang province, China & PWR & 15.2 & M & Early July & 18.93 \\
\hline 35 & Sha Hong Tao & Bud mutation from Kurakato Wase & Shaanxi province, China & PWR & 15.6 & M & Early July & 19.67 \\
\hline 36 & Zi Jin Hong 1 Hao & Natural seed cultivated by embryo rescue & Jiangsu, China & NYR & 9.7 & $\mathrm{~L}$ & Early June & 20.12 \\
\hline 37 & Ling Shen 1 Hao & Landrace & Zhejiang province, China & PWR & 17.9 & $\mathrm{H}$ & Early September & 20.38 \\
\hline 38 & Li You 5 Hao & unknown & Zhejiang province, China & NYR & 9.3 & $\mathrm{~L}$ & Late June & 20.89 \\
\hline 39 & Ling Shen 2 Hao & Landrace & Zhejiang province, China & PWR & 16.1 & $\mathrm{~L}$ & Early September & 21.11 \\
\hline 40 & Jin Xiu Huang Tao & Bai Hua $\times$ Yun Shu 1 & Shanghai, China & PYR & 15.1 & M & Early August & 21.14 \\
\hline 41 & Dong Feng Da Hong Tao & Landrace & Shanxi province, China & PWR & 12.58 & M & Middle June & 21.27 \\
\hline 42 & Meng Lu Shui Jing & Seedling & Zhejiang province, China & PWR & 15.5 & M & Middle July & 21.88 \\
\hline 43 & Zao Hong Tao & 60-4-1) ×Er Yuan Tao & China & PWR & 10.5 & $\mathrm{~L}$ & Early June & 21.90 \\
\hline 44 & Chun Mi & $\begin{array}{l}\text { 89-3-16 (Zao Hong } 2 \times \text { Li He Pan Tao }) \times \text { SD9238（Rui } \\
\text { Guang } 3 \times \text { May Fire })\end{array}$ & Henan province, China & PWR & 10.4 & M & Early June & 21.91 \\
\hline 45 & Xue Xiang Lu & Bai Hua $\times$ Chu Xiang Mei & Jiangsu province, China & PWR & 11.3 & M & Middle June & 22.09 \\
\hline 46 & Zhong Pan Tao 10 & Hong Shan Hu×91-4-8(NJN78×Feng Hua Pan Tao) & Henan province, China & PWR & 11.7 & M & Middle June & 22.59 \\
\hline 47 & Ying Guang You Tao & unknown & Zhejiang province, China & NWR & 12.6 & $\mathrm{M}$ & Late May & 23.42 \\
\hline
\end{tabular}




\begin{tabular}{|c|c|c|c|c|c|c|c|c|}
\hline 48 & Xia Cui & $\begin{array}{l}\text { Yu Hua } 2 \times 77-1-6((\text { Bai Hua } \times \text { Tachibana Wase }) \times \text { Zhao } \\
\text { Xia) }\end{array}$ & Jiangsu province, China & PWR & 12.4 & $\mathrm{M}$ & Late June & 23.95 \\
\hline 49 & Reddomun & Bai Feng $\times$ Bai Tao & Japan & PWR & 12.2 & M & Early July & 24.48 \\
\hline 50 & Da Guan 1 Hao & Selected from Nunomewase & Henan province, China & PWR & 9.3 & M & Early June & 24.81 \\
\hline 51 & $\mathrm{X} 1-4$ & Yu Lu×Hu Jing Mi Lu & Zhejiang province, China & PWR & 13.0 & $\mathrm{M}$ & Early July & 24.91 \\
\hline 52 & Kawanakajima Hakuto & $\begin{array}{l}\text { Found in White peach and Shang Hai Shui Mi mixed } \\
\text { garden }\end{array}$ & Japan & PWR & 15.1 & M & Early July & 24.94 \\
\hline 53 & Okubo & Seedling of Hakuho & Japan & PWR & 13.14 & $\mathrm{M}$ & Early July & 25.17 \\
\hline 54 & Okubo Late & Seedling of Okubo & Shanxi province, China & PWR & 13.14 & $\mathrm{H}$ & Middle July & 25.17 \\
\hline 55 & Xin Hong & Landrace & Zhejiang province, China & PWR & 14.1 & $\mathrm{M}$ & Early July & 25.20 \\
\hline 56 & Hu Jing Mi Lu & Seedling of Hakuho & Jiangsu province, China & PWR & 14.7 & $\mathrm{M}$ & Middle July & 25.43 \\
\hline 57 & Zao Feng Huang & unknown & Zhejiang province, China & PWR & 12.5 & $\mathrm{~L}$ & Middle June & 26.25 \\
\hline 58 & Yumyeong & $\begin{array}{l}\text { Da Hua Zao Sheng } \times \text { Bu Mu Zao Sheng or Okubo } \times \text { Bu } \\
\text { Mu Zao Sheng }\end{array}$ & Korea & PWR & 15.0 & $\mathrm{~L}$ & Middle July & 26.51 \\
\hline 59 & $\mathrm{Xin} Y u$ & Landrace & Zhejiang province, China & PWR & 14.9 & M & Middle July & 26.77 \\
\hline 60 & Springtime & Luken's Honey X July Elberta & USA & PWR & 10.0 & $\mathrm{M}$ & Early June & 26.88 \\
\hline 61 & Akatsuki & Bai Tao $\times$ Bai Feng & Japan & PWR & 12.8 & M & Early July & 27.26 \\
\hline 62 & $\mathrm{X} 1-7$ & Yu Lu×Hu Jing Mi Lu & Zhejiang province, China & PWR & 10.8 & M & Early July & 27.31 \\
\hline 63 & Mei Shuai & Okubo $\times 90-1($ Ba Yue Cui $\times$ Jing Yu) & Hebei province, China & PWR & 12.6 & M & Middle July & 27.67 \\
\hline 64 & Zhong You Tao 13 & unknown & Henan province, China & NYR & 12.1 & M & Early June & 27.95 \\
\hline 65 & Asama Hakuto & Bud mutation from Kouyou Hakuto & Japan & PWR & 16.2 & M & Middle July & 28.28 \\
\hline 66 & $\mathrm{Yu} \mathrm{Lu}$ & offspring of Shanghai Shumi & Zhejiang province, China & PWR & 15.5 & $\mathrm{H}$ & Late July & 28.36 \\
\hline 67 & Wasesimizu & Early variation of Shang Hai Shui Mi & / & PWR & 11.6 & $\mathrm{~L}$ & Middle June & 28.70 \\
\hline 68 & Mei Shuo & 'Jinyu' selfing & Hebei province, China & PWR & 12.3 & M & Early June & 29.17 \\
\hline 69 & Hakuho & Shanghai Cling & Japan & PWR & 12 & $\mathrm{M}$ & Middle July & 29.66 \\
\hline
\end{tabular}




\begin{tabular}{|c|c|c|c|c|c|c|c|c|}
\hline 70 & Hong Sha Zi & seedling & Shaanxi, China & PWR & 11.8 & M & Early June & 30.58 \\
\hline 71 & Sunago Wase & Seedling & Japan & PWR & 10.6 & M & Middle June & 31.50 \\
\hline 72 & Zhong You Tao 14 & 90-1-25[25-17(Jing Yu×NJN76) $\times$ Hake $] \times$ SD9238 & Henan province, China & PWR & 11.5 & M & Early June & 31.73 \\
\hline 73 & Qing Tao & Seedling & Zhejiang province, China & PWR & 17.7 & $\mathrm{H}$ & Early September & 32.34 \\
\hline 74 & Qin Wang & Seedling from Okubo & Shaanxi province, China & PWR & 17.1 & $\mathrm{~L}$ & Middle July & 33.45 \\
\hline 75 & Yan Hong & Seedling & Beijing, China & PWR & 15.9 & $\mathrm{~L}$ & Late July & 33.87 \\
\hline 76 & Wan Mi & seedling & Beijing, China & PWR & 14.7 & M & Early September & 34.08 \\
\hline 77 & Dong Feng Shui Mi & Landrace & Shanxi province, China & PWR & 9.6 & M & Middle June & 34.16 \\
\hline 78 & Nan Shan Tian Tao & Landrace & Guangdong province, China & PWR & 18.0 & $\mathrm{~L}$ & Middle July & 34.78 \\
\hline 79 & Qiu Bai Tao & Seedling & Zhejiang province, China & PWR & 13.9 & $\mathrm{~L}$ & Late July & 35.01 \\
\hline 80 & Jia Tang Tao & Landrace & Zhejiang province, China & PWR & / & $\mathrm{H}$ & Late July & 36.06 \\
\hline 81 & Zao Lu Pan Tao & Sa Hua Hong Pan Tao×Zao Xiang Yu & Beijing, China & PWR & 10.0 & M & Early June & 36.31 \\
\hline 82 & Nunome Wase & Seedling & Japan & PWR & 11.2 & $\mathrm{H}$ & Early June & 36.43 \\
\hline 83 & Yan Feng & Landrace & Zhejiang province, China & PWR & 14.3 & M & Middle June & 36.60 \\
\hline 84 & Jin Qiu & Yang Quan Rou Tao x Ming Xin & Shanxi province, China & PYR & 13.0 & $\mathrm{~L}$ & Middle August & 37.31 \\
\hline 85 & Mei Jin & 'Jinyu' selfing & Hebei province, China & PYR & 14.6 & $\mathrm{~L}$ & Early July & 37.42 \\
\hline 86 & Zao Zhen Bao & unknown & Zhejiang province, China & PWR & 10.3 & M & Late June & 39.84 \\
\hline 87 & Yuan Meng & Hu Jing Mi Lu×Hakuri & Zhejiang province, China & PWR & 13.1 & $\mathrm{~L}$ & Early August & 40.81 \\
\hline 88 & Kurakato Wase & & Japan & PWR & 10.0 & $\mathrm{H}$ & Middle June & 41.33 \\
\hline 89 & Tai Yuan Shui Mi & Landrace & Shanxi province, China & PWR & 17.4 & $\mathrm{~L}$ & Early August & 41.66 \\
\hline 90 & Qiu Fen & Landrace & Shanxi province, China & PWR & 20.5 & $\mathrm{H}$ & Late July & 42.54 \\
\hline 91 & Qiu Xiang & Seedling & Beijing, China & PWR & 12.3 & $\mathrm{H}$ & Early September & 42.60 \\
\hline 92 & Hong Bu Ruan & Landrace & Shanxi province, China & PWR & 15.5 & M & Late July & 43.35 \\
\hline 93 & Mei Gui Lu & Sunago Wase $\times$ Yu Hua Lu & Zhejiang province, China & PWR & 11.9 & M & Early June & 44.86 \\
\hline 94 & Zhong Hua Shou Tao & Selection and breeding from the bud mutation of winter & Shandong,China & PWR & 12.2 & $\mathrm{H}$ & Early September & 47.76 \\
\hline
\end{tabular}




\begin{tabular}{|c|c|c|c|c|c|c|c|c|}
\hline & & peach in north China & & & & & & \\
\hline 95 & Zheng Huang 3 Hao & Zao Shu Huang Gan $\times$ Feng Huang & Henan province, China & PYR & 6.2 & M & Early July & 49.01 \\
\hline 96 & Wan Bai Mi & Wu Yun $\times$ Bai Feng & Jiangsu province, China & PWR & 14.0 & $\mathrm{H}$ & Early September & 49.22 \\
\hline 97 & Yu Lu Pan Tao & Landrace & Shanghai, China & PWF & 15.0 & $\mathrm{H}$ & Middle July & 49.92 \\
\hline 98 & Taiyuan Shui Mi & Seedling & Shanxi province, China & PWR & 10.5 & M & Middle June & 50.90 \\
\hline 99 & Jing $\mathrm{Yu}$ & Okubo $\times$ Xingjin You Tao & Beijing, China & PWR & 14.4 & $\mathrm{~L}$ & Middle July & 55.09 \\
\hline 100 & Jin Shuo & Yingqing $\mathrm{x}$ Yangtao & Shanghai, China & PYR & 17.5 & $\mathrm{H}$ & Early September & 57.89 \\
\hline 101 & Jin Hua & Seedling of Jin Xiu & Shanghai, China & PYR & 17.3 & M & Late August & 60.06 \\
\hline 102 & Qing Feng & Okubo $\times$ Xin Duan Yang & Beijing, China & PYR & 14.6 & $\mathrm{H}$ & Middle August & 60.44 \\
\hline 103 & F2-18 & Hu Jing Mi Lu×Hakuri & Zhejiang province, China & PYR & 15.0 & $\mathrm{H}$ & Early July & 64.41 \\
\hline
\end{tabular}

*The first letter refers to P-peach, N-Nectarine; the second letter refers to flesh color: W-white, Y-Yellow flesh, R-Red flesh; the third letter refers to fruit shape: F-Flat, R-round shape 
Supplementary Table 2. Pru p 3 content of peel and pulp in 24 peach varieties.

\begin{tabular}{lllccl}
\hline \multirow{2}{*}{ Varieties } & \multirow{2}{*}{ Origin } & \multirow{2}{*}{ Characteristics } & \multicolumn{3}{c}{ Pru p 3, mean \pm SD, $\mu$ g/g of fresh weight } \\
\cline { 3 - 5 } & & & Pulp & Peel & Whole fruit \\
\hline May fire & USA & NYR & $0.50 \pm 0.10$ & $6.43 \pm 0.77$ & $3.54 \pm 0.17$ \\
Xue Bu Dai & China & PRR & $0.77 \pm 0.11$ & $9.58 \pm 0.01$ & $4.00 \pm 0.95$ \\
Hu You 278 & China & NYR & $0.34 \pm 0.20$ & $7.63 \pm 1.09$ & $4.02 \pm 0.84$ \\
Armking & USA & NYR & $0.86 \pm 0.29$ & $10.49 \pm 0.03$ & $6.68 \pm 1.50$ \\
Zao Hong Zhu & China & NWR & $0.63 \pm 0.01$ & $29.9 \pm 3.81$ & $7.41 \pm 0.55$ \\
Zhong Nong Jin Hui & China & NYR & $0.26 \pm 0.01$ & $33.57 \pm 0.66$ & $8.02 \pm 0.66$ \\
Chao Li Chun & China & NYR & $1.88 \pm 0.54$ & $52.22 \pm 0.69$ & $13.53 \pm 0.09$ \\
Spring Snow & USA & PWR & $1.25 \pm 1.24$ & $63.24 \pm 6.03$ & $14.84 \pm 1.96$ \\
Hu You 018 & China & NYR & $0.20 \pm 0.01$ & $77.25 \pm 5.52$ & $17.64 \pm 0.32$ \\
Hang Mi Yi Hao & China & PWR & $0.58 \pm 0.00$ & $83.04 \pm 9.27$ & $18.93 \pm 0.54$ \\
Ying Guang You Tao & China & NWR & $2.54 \pm 0.04$ & $111.7 \pm 5.26$ & $23.42 \pm 0.91$ \\
Xia Cui & China & PWR & $2.62 \pm 0.67$ & $122.25 \pm 5.04$ & $23.95 \pm 1.96$ \\
Kawanakajima Hakuto & Japan & PWR & $2.56 \pm 0.05$ & $145.0 \pm 11.3$ & $24.94 \pm 0.64$ \\
Hu Jing Mi Lu & China & PWR & $3.08 \pm 0.14$ & $152.34 \pm 6.46$ & $25.43 \pm 1.22$ \\
Xin Yu & China & PWR & $3.95 \pm 1.23$ & $135.65 \pm 6.95$ & $26.77 \pm 6.17$ \\
Zao Lu Pan Tao & China & PWR & $3.91 \pm 0.05$ & $162.10 \pm 3.86$ & $36.31 \pm 0.84$ \\
Nunome Wase & Japan & PWR & $4.27 \pm 0.13$ & $168.15 \pm 14.75$ & $36.43 \pm 1.33$ \\
Mei Jin & China & PYR & $4.78 \pm 1.85$ & $143.00 \pm 0.02$ & $37.42 \pm 1.78$ \\
Kurakato Wase & Japan & PWR & $2.86 \pm 0.63$ & $171.47 \pm 2.51$ & $41.33 \pm 0.21$ \\
Mei Gui Lu & China & PWR & $4.75 \pm 0.09$ & $179.26 \pm 9.54$ & $44.86 \pm 2.12$ \\
Zheng Huang 3 Hao & China & PYR & $5.41 \pm 2.84$ & $198.48 \pm 3.74$ & $49.01 \pm 5.01$ \\
Yu Lu Pan Tao & China & PWF & $7.26 \pm 0.25$ & $196.72 \pm 3.28$ & $49.92 \pm 7.07$ \\
Jin Shuo & China & PYR & $7.14 \pm 0.28$ & $199.4 \pm 4.06$ & $57.89 \pm 3.50$ \\
Jin Hua & China & PYR & $9.52 \pm 0.18$ & $211.39 \pm 13.10$ & $60.06 \pm 6.19$ \\
\hline
\end{tabular}

'Xue Bu Dai', 'Hu You 278', 'Mei Jin' and 'Jin Shuo' were used for Immuno-cytochemical localization; 'Hu You 278' and 'Mei Jin' were also tested for SPT. Two Flat peach 'Zao Lu Pan Tao' and 'Yu Lu Pan Tao' have high Pru p 3 content. 